\title{
The Fer tyrosine kinase regulates interactions of Rho GDP-Dissociation Inhibitor $\alpha$ with the small GTPase Rac
}

Fei Fei ${ }^{1+}$, Soo-Mi Kweon ${ }^{1,2+}$, Leena Haataja ${ }^{3}$, Paulo De Sepulveda ${ }^{4}$, John Groffen ${ }^{1,5}$, Nora Heisterkamp ${ }^{1,5^{*}}$

\begin{abstract}
Background: RhoGDI proteins are important regulators of the small GTPase Rac, because they shuttle Rac from the cytoplasm to membranes and also protect Rac from activation, deactivation and degradation. How the binding and release of Rac from RhoGDI is regulated is not precisely understood.

Results: We report that the non-receptor tyrosine kinase Fer is able to phosphorylate RhoGDl $\alpha$ and form a direct protein complex with it. This interaction is mediated by the C-terminal end of RhoGDla. Activation of Fer by reactive oxygen species caused increased phosphorylation of RhoGDl $\alpha$ and pervanadate treatment further augmented this. Tyrosine phosphorylation of RhoGDl $\alpha$ by Fer prevented subsequent binding of Rac to RhoGDl $\alpha$, but once a RhoGDla-Rac complex was formed, the Fer kinase was not able to cause Rac release through tyrosine phosphorylation of preformed RhoGDla-Rac complexes.
\end{abstract}

Conclusions: These results identify tyrosine phosphorylation of RhoGDla by Fer as a mechanism to regulate binding of RhoGDla to Rac.

\section{Background}

Rac proteins belong to the larger family of Rho GTPases that act as molecular switches. When bound to GTP, they are in an active conformation. Hydrolysis of GTP to GDP causes an inactivating conformational change. Racs are post-translationally modified by the removal of $\mathrm{C}$-terminal amino acid residues, followed by the attachment of a lipid moiety in the form of a geranyl-geranyl group [1].

Because of this modification, mature Rac proteins are lipophilic and are only present in the cytosol when bound to the chaperone and transport protein RhoGDI. RhoGDI regulates the movement of Rac between the cytosol and membranes. Moreover, a recent study showed that Rho proteins compete for binding to a limited pool of RhoGDI, which, once it has bound them, provides stabilization and protection from degradation [2].

\footnotetext{
* Correspondence: heisterk@hsc.usc.edu

† Contributed equally

${ }^{1}$ Section of Molecular Carcinogenesis, Division of Hematology/Oncology, The Saban Research Institute of Childrens Hospital Los Angeles, CA 90027, USA
} Full list of author information is available at the end of the article
There are only three distinct RhoGDI proteins, of which RhoGDI $\alpha$ is the most ubiquitously expressed. The structure of RhoGDI bound to small GTPases has been elucidated. Rac makes contact with both $\mathrm{N}$-and C-terminal regions in RhoGDI, with its hydrophobic tail fitting into a pocket in the C-terminal part of RhoGDI. However, the exact mechanism by which binding and release of Racs from RhoGDI is mediated is not well known $[3,4]$.

RhoGDI can be phosphorylated on serine S101 and S174 by the serine/threonine kinase Pak [5] and PKC $\alpha$ also phosphorylates RhoGDI $\alpha$, on residue S96 [6]. Interestingly, constitutively activated oncogenic Src, which has a deregulated tyrosine kinase activity, was shown to phosphorylate RhoGDI $\alpha$ on tyrosine residue Y156 [7]. In the current study we report that a normal cellular tyrosine kinase which is member of the Src family, Fer, is able to specifically tyrosine phosphorylate RhoGDIa and that this can regulate the binding to Rac1.

\section{Results and discussion}

The $B C R / A B L$ oncogene encodes a chimeric protein that contains most of the Abl tyrosine kinase. Proteomic 
analysis of cells transfected with $\mathrm{Bcr} / \mathrm{Abl}$ [8] reported changes in phosphorylation of RhoGDI. To investigate if the deregulated Bcr/Abl tyrosine kinase, similar to oncogenic Src [7], is able to modify RhoGDI $\alpha$, we performed an immunocomplex kinase assay with cells expressing $\mathrm{Bcr} / \mathrm{Abl}$ or with Fer, a non-receptor tyrosine kinase related to $\mathrm{Abl}$, in the presence of added recombinant GST-RhoGDI $\alpha$ or control GST. As shown in Figure 1A, left panel, the Bcr/Abl protein exhibited strong autophosphorylation activity, as expected. However, there was very little phosphorylated RhoGDI $\alpha$. In contrast, there was a prominent signal of phosphorylated RhoGDI $\alpha$ in the sample expressing Fer (Figure 1A right panel).

To examine whether Fer and RhoGDI $\alpha$ interact in cells, we transfected COS-1 cells with pEGFP-Fer (wild type, WT) or kinase dead D742R mutant pEGFP-Fer (mutant, MT), with or without RhoGDI $\alpha$ and investigated possible protein complex formation between these by co-immunoprecipitation. As shown in Figure 1B, left panel, Fer-WT clearly co-immunoprecipitated with RhoGDI $\alpha$, which was phosphorylated on tyrosine, as judged by Western blotting using phosphotyrosine antibodies. In the converse experiment (Figure 1B, right panel, Fer IP) we confirmed the interaction between Fer-WT and RhoGDI $\alpha$. In the presence of the Fer mutant lacking tyrosine kinase activity, no tyrosine phosphorylated RhoGDI $\alpha$ was detected, indicating that Fer tyrosine kinase activity is directly or indirectly needed to generate tyrosine phosphorylated RhoGDI $\alpha$. Interestingly, the kinase-defective Fer protein exhibited a reduced ability to form a complex with RhoGDI $\alpha$ (compare lanes in IP Fer, IB for RhoGDI $\alpha$ ), suggesting that the binding of these two proteins is facilitated by tyrosine phosphorylation. This is in agreement with the finding that the isolated Fer $\mathrm{SH} 2$ domain binds to RhoGDI $\alpha$ on far-Western blots (not shown). Importantly, we also were able to co-immunoprecipitate endogenous Fer with endogenous RhoGDI $\alpha$ in a lysate of the mast cell line RBL-2H3. This indicates that the complex formation between RhoGDI $\alpha$ and Fer also takes place without overexpression (Figure 1C).

Although reactive oxygen species (ROS) were originally identified as a bactericidal mechanism, a role for $\mathrm{H}_{2} \mathrm{O}_{2}$ as secondary messenger has now been wellestablished. Many groups suggest that Src may be a primary target of ROS, with some studies reporting activation of Src kinase activity upon ROS exposure [9]. Since constitutively activated oncogenic Src had been shown to phosphorylate RhoGDI $\alpha[10,11]$, we tested if exposure of $\mathrm{c}$-Src to $\mathrm{H}_{2} \mathrm{O}_{2}$ would activate it to phosphorylate RhoGDI $\alpha$. As shown in Figure 2A, in the presence of c-Src and after $\mathrm{H}_{2} \mathrm{O}_{2}$ exposure, we could detect tyrosine phosphorylated RhoGDI $\alpha$. Sangrar et al [12] showed that ROS also induce Fer tyrosine autophosphorylation as well as trans-phosphorylation activity. As shown in Figure $2 \mathrm{~B}$, we confirmed Fer activation by $\mathrm{H}_{2} \mathrm{O}_{2}$ in different types of cells by immunoprecipitation and Western blotting with anti-phosphotyrosine antibodies. In addition, when we co-transfected Fer with RhoGDI $\alpha$ and treated the cells with $\mathrm{H}_{2} \mathrm{O}_{2}$, increased RhoGDI $\alpha$ tyrosine phosphorylation was detected (Figure 2C). The tyrosine phosphorylated RhoGDI $\alpha$ was visible as two discrete bands, indicating this protein is subject to processive phosphorylation.

Tyrosine phosphorylation of signal transduction proteins in the absence of an oncogenic, constitutively activated tyrosine kinase may be difficult to detect due to the transient nature of the phosphorylation and the activity of phosphatases. Wang et al. reported that pervanadate treatment allows detection of phosphorylation on RhoGDI $\alpha$ because this inhibits protein-tyrosine phosphatases and traps proteins in their tyrosine-phosphorylated form [13]. Next, we examined if pervanadate would further activate Fer and increase RhoGDI $\alpha$ tyrosine phosphorylation. As shown in Figure 3A, when cells co-expressing RhoGDI $\alpha$ and Fer were exposed to pervanadate for 5 minutes, a marked increase in tyrosine phosphorylation was seen both in Fer and RhoGDI $\alpha$ proteins (Figure 3A). The N-terminal regulatory domain of RhoGDI binds to the switch region of Rac whereas the C-terminal end adopts a conformation that accommodates the hydrophobic tail of Rac [6]. To examine which domain interacts with Fer, we expressed the $\mathrm{N}$ - and C-terminus of RhoGDI $\alpha$ as Xpress-tagged proteins, together with Fer, and immunoprecipitated each fragment of RhoGDI $\alpha$ with Xpress antibodies. We could not detect a clear interaction of the $\mathrm{N}$-terminal end with Fer (data not shown). As shown in Figure 3B (RhoGDI $\alpha-C T+F e r)$, in immunoprecipitates with the C-terminal end of RhoGDI $\alpha$ and in the presence of pervanadate, we recovered tyrosine-phosphorylated Fer. Moreover, this domain of RhoGDI $\alpha$ also was tyrosine phosphorylated (Figure 3B). This indicates that Fer interacts with and tyrosine phosphorylates residues in the part of RhoGDI $\alpha$ that includes the domain that binds to the C-terminal end of Rac. We were also able to demonstrate that endogenous RhoGDI $\alpha$ was phosphorylated on tyrosine in response to pervanadate, which activates Fer in RBL-2H3 cells (Figure 3C).

We used purified proteins to examine if tyrosine phosphorylation of RhoGDI $\alpha$ could regulate its ability to bind Rac. Fer was expressed as a GST fusion protein in baculovirus. In Figure 4A (left panel), we first incubated GSTRhoGDI $\alpha$ with Rac to allow these proteins to form a complex. Then GST-Fer or control GST was added with a kinase reaction buffer and the kinase reaction was initiated. As shown in the top panel (pY20 blot), both autophosphorylation of Fer and transphosphorylation of 


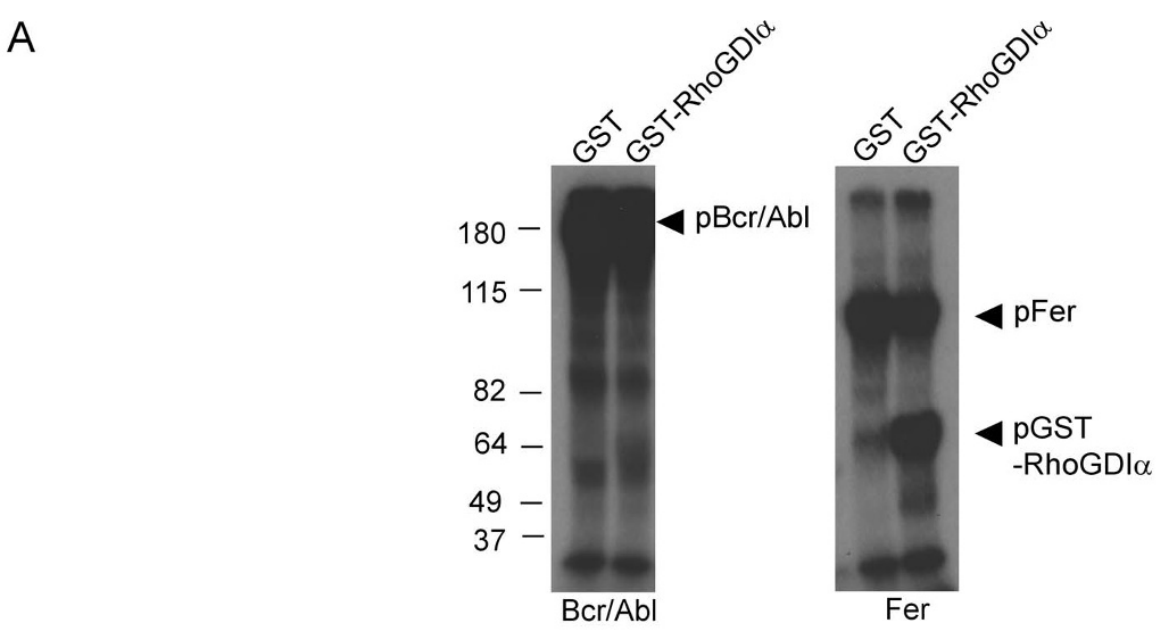

\section{B}
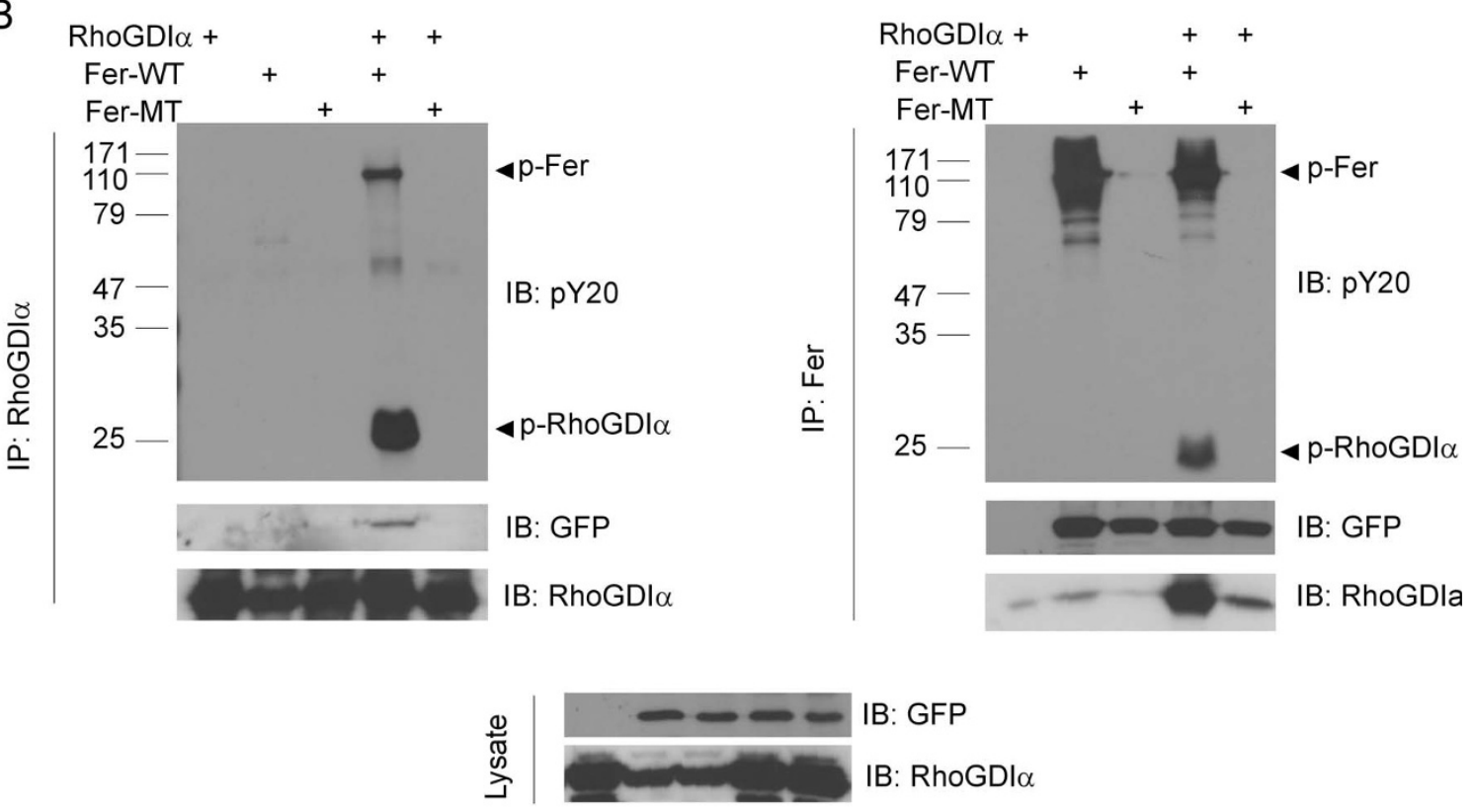

IB: GFP

IB: RhoGDl $\alpha$

C

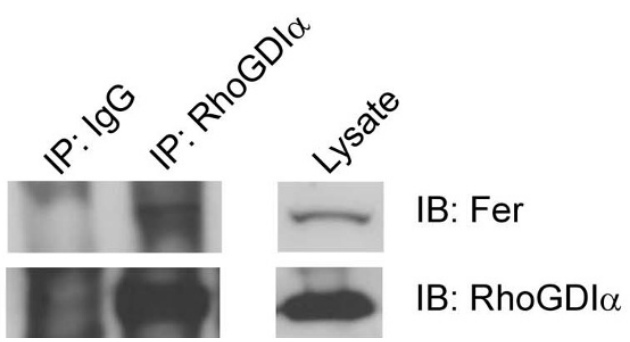

Figure 1 Fer forms a complex with RhoGDI $\alpha$ and phosphorylates it on tyrosine. (A) COS-1 cells were transfected with Bcr/Abl or Fer (pSG5-Fer). Autoradiogram of immune complex kinase reaction performed with immunoprecipitated Bcr/Abl or Fer (CH-6 antibodies) as indicated, with bacterially expressed GST-RhoGDl $\alpha$ or control GST added. (B) Immunoprecipitation (IP) from cells transfected with pEGFP taggedFer-WT or Fer-MT alone or together with RhoGDl $\alpha$ using RhoGDl $\alpha$ antibodies (Santa Cruz) (left panel) or Fer monoclonal rat antiserum (right panel). Antibodies used for immunoblot (IB) are indicated below the panels. After reaction with the pY20 antibodies, blots were stripped and reacted with RhoGDla (Santa Cruz) or GFP antibodies. Arrowheads point to the locations of Fer and RhoGDla. Molecular weight standards are in kDa. (C) Co-immunoprecipitation of endogenous Fer (using antibodies from Abcam) and RhoGDla (Santa Cruz antibodies) proteins in RBL-2H3 mast cell lysates. These results are representative of three independent experiments. 


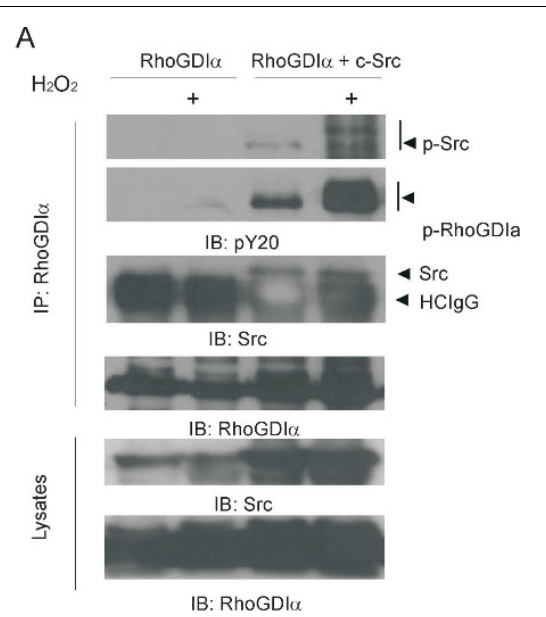

B
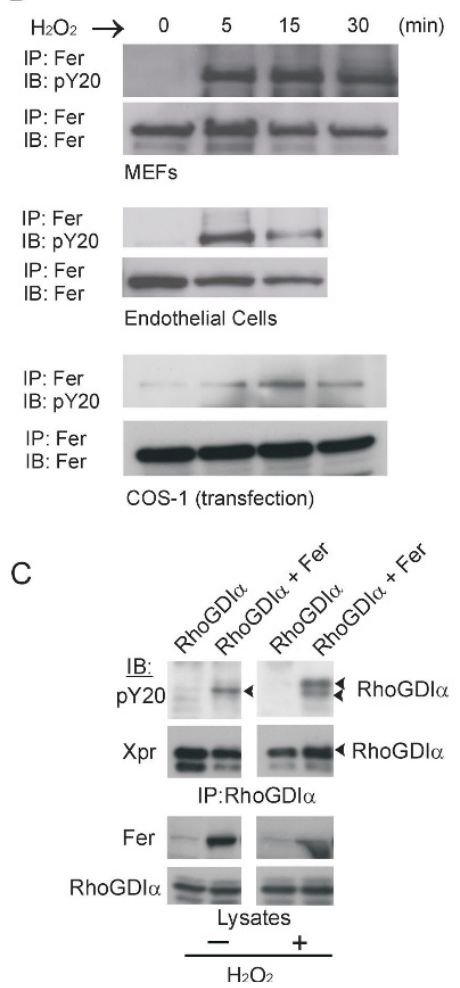

Figure 2 Activation of Fer and non-oncogenic Src. (A) RhoGDla was expressed alone or with c-Src as indicated above the panel. Cells in lanes marked with a " + " were stimulated with $\mathrm{H}_{2} \mathrm{O}_{2}(10 \mathrm{mM})$ for 15 minutes and RhoGDla was immunoprecipitated. Antiphosphotyrosine (pY20) blots were stripped and re-probed with RhoGDla antibodies (Santa Cruz). (B) Mouse embryonic fibroblasts (MEFs), mouse endothelial cells and cells transfected with Fer were treated with $5 \mathrm{mM}$ of $\mathrm{H}_{2} \mathrm{O}_{2}$ for the indicated time. Fer protein was immunoprecipitated from the lysates followed by a pY20 immunoblot to detect activated tyrosine phosphorylated Fer (Abcam). (C) COS-1 cells transfected with Xpress-tagged RhoGDla were stimulated or not with $\mathrm{H}_{2} \mathrm{O}_{2}$, as indicated below the panels. RhoGDl $\alpha$ antibodies (Upstate Biotechnology) were used for immunoprecipitation. Antibodies used for Western blotting are indicated to the left of the panels. Fer, $\mathrm{CH}-6$ antibodies. This result is representative of two independent experiments.
RhoGDI $\alpha$ occurred as expected. However, there was no evidence that Rac was tyrosine phosphorylated (not shown). Rac protein was recovered in immunoprecipitates from both Fer-treated and non-Fer treated samples, indicating that the presence or absence of Fer does not affect preformed Rac-RhoGDI $\alpha$ complexes. A different result was obtained when Fer was first incubated with RhoGDI $\alpha$ and a kinase reaction was done. When Rac was subsequently added and allowed to bind, we found that it was not co-immunoprecipitated with RhoGDI $\alpha$ in the Fer-treated sample (Figure 4B). The lack of binding of Rac to RhoGDI $\alpha$ could be because tyrosine-phosphorylated RhoGDI $\alpha$ has decreased affinity for Rac, or because the binding of Fer to RhoGDI $\alpha$ prevented subsequent binding of Rac to RhoGDI $\alpha$. Interestingly, this effect is similar to that reported for oncogenic Src: when Src phosphorylated RhoGDI $\alpha$, the affinity of RhoGDI $\alpha$ for Rac was also significantly reduced [7].

\section{Conclusions}

Tyrosine phosphorylation as a mechanism to regulate RhoGDIa has not been widely investigated, although it was recently reported that pervanadate treatment of a pancreatic islet beta cell line caused the appearance of phosphotyrosine on RhoGDI $\alpha$ and was accompanied by lack of Cdc42 binding [13]. There is also increasing evidence that $\mathrm{H}_{2} \mathrm{O}_{2}$ regulates the balance of tyrosine phosphorylation and de-phosphorylation in cells. The current study shows that non-oncogenic tyrosine kinases are likely to play a role in regulating the binding of Rac GTPases to RhoGDI $\alpha$ and through this, the cellular pool of Rac.

\section{Methods}

\section{Antisera and DNA constructs}

The Fer polyclonal rabbit anti-Fer $\mathrm{CH}-6$ antiserum $[14,15]$ and anti-Fer monoclonal rat antiserum [16] have been previously described and were used for immunoprecipitation. RhoGDI $\alpha$ antibodies were obtained from Upstate Biotechnology (Temecula, CA) or Santa Cruz (Santa Cruz, CA). Antibodies against Fer, GFP, Xpress and GST were from Abcam (San Francisco, CA), Santa Cruz, Invitrogen (Carlsbad, CA) and Santa Cruz, respectively. Antibodies against pY20 and Rac1 were purchased from BD Bioscience (Franklin Lakes, NJ). pSG5-Fer was constructed by subcloning a $2.9 \mathrm{~kb}$ BamHI fragment from human Fer clone L3, encompassing the entire coding region and small 5 ' and 3 ' untranslated regions, into the BamHI site of pSG5. pEGFP-Fer (WT), pEGFP-Fer (MT D742R) and RhoGDI $\alpha$ have been previously described $[17,18]$. The $\mathrm{N}$ - and C-terminal fragments of RhoGDI $\alpha$ were subcloned from pEGFP-RhoGDI $\alpha$-NT and pEGFP-RhoGDI $\alpha-C T$ into pcDNA3.1/HisB by digestion with EcoRI/XbaI and have 


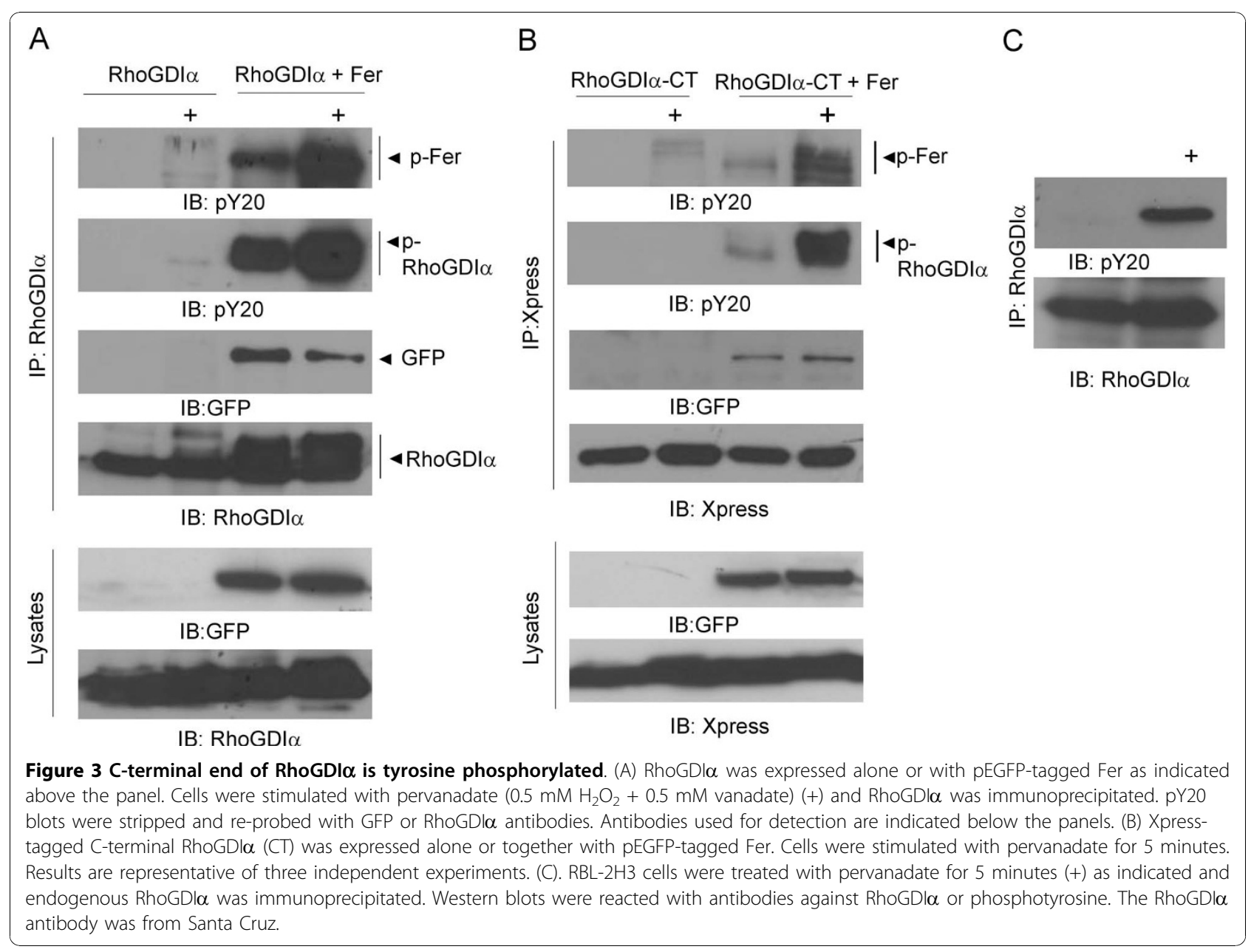

been previously described [18]. p-LNCX mouse c-Src was obtained from Addgene (Cambridge, MA).

\section{Recombinant proteins}

A fragment containing the start codon of human Fer (5'-ggggatccatggggtttgggagtgacctg) was used to introduce a BamHI site immediately 5' to the Fer ATG. A 155 bp fragment amplified with this primer and a 3' oligonucleotide was digested with BamHI $\times \mathrm{NsiI}$ and the $5^{\prime}$ BamHINsiI fragment ligated with a 3' $1.2 \mathrm{~kb}$ NsiI-KpnI fragment isolated from plasmid Fer SV-7-2 [19] into pSK digested with BamHI $\times$ KpnI. A $1.4 \mathrm{~kb}$ BamHI-KpnI fragment from this plasmid was ligated with a $1.3 \mathrm{~kb}$ KpnI-EcoRI fragment into the pAcG2T baculovirus transfer vector digested with BamHI $\times$ EcoRI. S. frugiperda Sf9 cells were grown in Grace's Insect medium (Invitrogen) with 10\% FBS (heat-inactivated) and $10 \mu \mathrm{g} /$ ml Gentamycin (Invitrogen). DNA transfections used 0.5 $\mu \mathrm{g}$ BaculoGold viral DNA and $2 \mu \mathrm{g}$ of recombinant baculovirus transfer vector. Three rounds of viral amplification were performed and the third amplification used for large-scale infection and baculovirus production. GST fusion proteins were purified by glutathione-agarose. GST-Fer was washed and concentrated using a Centricon-10 (Amicon) filter. Bacterially expressed RhoGDI $\alpha$ and Rac1 were prepared as previously described, with the GST tag removed from the Rac1 protein by thrombin cleavage [18].

\section{Cell lines and immunoprecipitations}

COS-1 cells and rat basophilic leukemia/mast cell RBL$2 \mathrm{H} 3$ cells were obtained from the ATCC (Rockville, MD). All tissue culture media and supplements were from Invitrogen (Carlsbad, CA). COS-1 cells were cultured in DMEM or RPMI containing $10 \%$ fetal bovine serum (FBS), penicillin $(100 \mathrm{U} / \mathrm{ml})$, streptomycin $(100 \mu \mathrm{g} / \mathrm{ml})$, L-glutamine and sodium pyruvate $(1 \mathrm{mM})$. RBL-2H3 cells were cultured in MEM containing 15\% FBS, $1 \%$ penicillin/streptomycin. Immunoprecipitations and immunoblotting were performed as described before [18].

\section{Immunocomplex kinase assay}

The kinase activity of Fer or Bcr/Abl was measured as described previously [18]. Briefly, Fer-transfected COS-1 


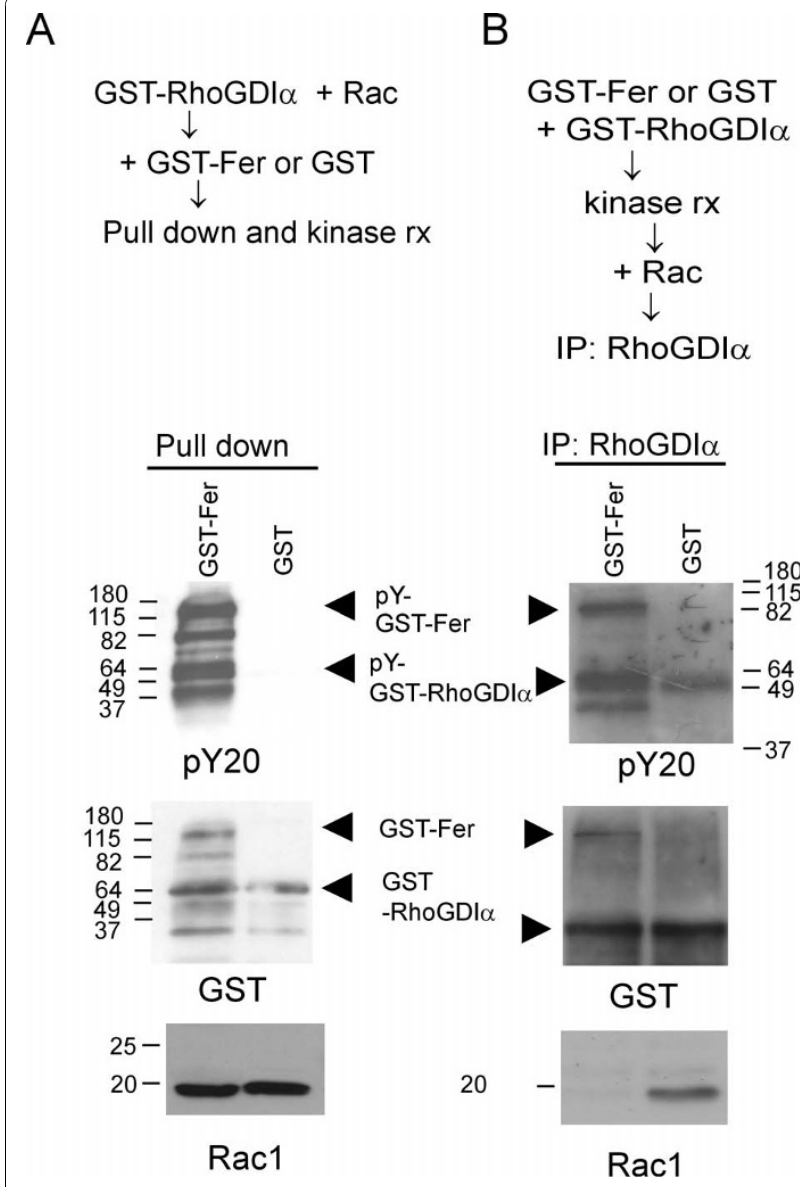

Figure 4 Fer interferes with binding of RhoGDI $\alpha$ and Rac. (A) GST-RhoGDl $\alpha$ was pre-incubated with Rac, after which GST-Fer (GFer) or GST (G) was added and GST fusion proteins were pulled down with glutathione agarose, followed by kinase reactions. (B) GST-RhoGDla was pre-incubated with GST-Fer or GST in kinase buffer, after which Rac was added and RhoGDla was immunoprecipitated. 50 pmol of RhoGDl $\alpha$ and Rac1, with 25 pmol of GST and GST-Fer were used. Blots were reacted with Rac1 antibodies or pY20, then stripped and reacted with GST antibodies. This result is representative of two independent experiments.

cells were lysed with $1 \times$ RIPA buffer $(50 \mathrm{mM}$ Tris$\mathrm{HCl}, \mathrm{pH} 8.0,150 \mathrm{mM} \mathrm{NaCl}, 1 \%$ Igepal, $0.5 \%$ deoxycholate, $0.1 \%$ SDS, $5 \mathrm{mM}$ EDTA) containing PMSF, leupeptin, and aprotinin and then incubated with Fer antibody, $\mathrm{CH}-6$ for $2 \mathrm{~h}$ at $4{ }^{\circ} \mathrm{C}$. After incubation with protein A agarose for $30 \mathrm{~min}$ at $4^{\circ} \mathrm{C}$, the affinity complex was washed with ice-cold high salt HNTG buffer (80 mM HEPES, pH 7.5, 0.1\% Triton-X100, $500 \mathrm{mM}$ $\mathrm{NaCl}$ and $10 \%$ glycerol) and then with ice-cold low salt HNTG buffer (80 mM HEPES, pH 7.5, 0.1\% Triton$\mathrm{X} 100,150 \mathrm{mM} \mathrm{NaCl}$ and $10 \%$ glycerol). For kinase reactions, $10 \mu \mathrm{l}$ of $40 \mathrm{mM}$ PIPES and $10 \mu \mathrm{l}$ of $40 \mathrm{mM}$ $\mathrm{MnCl}_{2}$ were added as well as $0.5 \mu \mathrm{g}$ of GST-RhoGDI $\alpha$ protein or GST protein. The reactions were initiated by addition of $10 \mu \mathrm{Ci}$ of $\left[\gamma^{32} \mathrm{P}\right] \mathrm{ATP}(5000 \mathrm{Ci} / \mathrm{mmol})$ (Amersham). After $40 \mathrm{~min}$ on ice, reactions were terminated by the addition of $2 \times$ SDS-sample buffer and boiling for $5 \mathrm{~min}$. Samples were run on 8\% SDS-PAGE gels and the radioactively labeled proteins visualized by autoradiography.

\section{In vitro binding assay for interaction of RhoGDI $\alpha$ and Rac1}

To investigate possible binding of phosphorylatedRhoGDI $\alpha$ to Rac1, GST-RhoGDI $\alpha$ protein $(50 \mathrm{pmol})$ was first combined with GST-Fer $(25 \mathrm{pmol})$ or GST alone $(25 \mathrm{pmol})$ in $100 \mu \mathrm{l}$ of kinase buffer $(20 \mathrm{mM}$ HEPES, pH 7.5, $50 \mathrm{mM} \mathrm{NaCl}, 25 \mathrm{mM} \mathrm{MnCl} 2,10 \mathrm{mM}$ $\mathrm{MgCl}_{2}, 1 \mathrm{mM}$ DTT). $20 \mu \mathrm{M}$ of ATP was added to the mixture, which was incubated for $30 \mathrm{~min}$ at $4^{\circ} \mathrm{C}$ for the kinase reaction. Next, $400 \mu \mathrm{l}$ of binding buffer $(1 \times$ DPBS, $0.1 \%$ IgePal, $10 \%$ glycerol, $0.5 \mathrm{mM}$ DTT containing the protease inhibitors PMSF, leupeptin, and aprotinin) and 50 pmol Rac1 protein were added, followed by incubation for $1 \mathrm{~h}$ at $4^{\circ} \mathrm{C}$. Incubation with antibody against RhoGDI $\alpha$ for $1 \mathrm{~h}$ at $4^{\circ} \mathrm{C}$ was followed by Protein $\mathrm{A}$ agarose addition and a second incubation $1 \mathrm{~h}$ at $4^{\circ} \mathrm{C}$. To investigate if phosphorylation of RhoGDI $\alpha$ by Fer releases Rac1, GST-RhoGDI $\alpha$ protein was incubated with Rac1 in binding buffer for $1 \mathrm{~h}$ at $4^{\circ} \mathrm{C}$; GST-Fer or GST alone was added, and a pull down with glutathione agarose was done, followed by washing of the precipitate. Kinase reactions were initiated by addition of kinase buffer and ATP for $30 \mathrm{~min}$ at $4^{\circ} \mathrm{C}$.

\section{List of abbreviations}

RhoGDI: Rho GDP-dissociation inhibitor; ROS: reactive oxygen species

\section{Acknowledgements}

We thank Konstadinos Moissoglu and Martin Schwartz (University of Virginia) for the generous gift of the pEGFP-RhoGDla NT and CT clones; and Toshiki Itoh and Tadaomi Takenawa (Kobe University Graduate School Medicine, Japan) for providing pEGFP-Fer-WT and pEGFP-FerD742R clones. Johanna ten Hoeve is acknowledged for the construction of pSG5-Fer. This work was supported by PHS grants HL071945 (JG) and HL060231 (JG, NH).

\section{Author details}

${ }^{1}$ Section of Molecular Carcinogenesis, Division of Hematology/Oncology, The Saban Research Institute of Childrens Hospital Los Angeles, CA 90027, USA. ${ }^{2}$ Leukemia Research Program, Childrens Hospital Los Angeles, CA 90027, USA. ${ }^{3}$ Metabolism, Endocrinology \& Diabetes, University of Michigan, Ann Arbor, MI 48105, USA. ${ }^{4}$ INSERM, UMR 891, Centre de Recherche en Cancérologie de Marseille, Laboratoire de Signalisation, Hématopoïèse et Mécanismes de l'Oncogenèse, Marseille, France. ${ }^{5}$ Department of Pathology, Keck School of Medicine, University of Southern California, Los Angeles, CA 90033, USA.

\section{Authors' contributions}

FF performed experiments, brought in new ideas and wrote part of the manuscript; S-M K performed experiments and wrote part of the manuscript LH made the baculovirus Fer protein; PS provided critical reagents; JG designed experiments and provided critical input; $\mathrm{NH}$ provided ideas and wrote the manuscript. All authors read and approved the final manuscript. 


\section{References}

1. Ridley AJ: Rho GTPases and actin dynamics in membrane protrusions and vesicle trafficking. Trends Cell Biol 2006, 16:522-529.

2. Boulter E, Garcia-Mata R, Guilluy C, Dubash A, Rossi G, Brennwald PJ, Burridge K: Regulation of Rho GTPase crosstalk, degradation and activity by RhoGDI1. Nat Cell Biol 2010, 12:477-483.

3. Dovas A, Couchman JR: RhoGDI: multiple functions in the regulation of Rho family GTPase activities. Biochem J 2005, 390(Pt 1):1-9.

4. Dransart E, Olofsson B, Cherfils J: RhoGDIs revisited: novel roles in Rho regulation. Traffic 2005, 6:957-966.

5. DerMardirossian C, Schnelzer A, Bokoch GM: Phosphorylation of RhoGDI by Pak1 mediates dissociation of Rac GTPase. Mol Cell 2004, 15:117-127.

6. Knezevic N, Roy A, Timblin B, Konstantoulaki M, Sharma T, Malik AB, Mehta D: GDI-1 phosphorylation switch at serine 96 induces RhoA activation and increased endothelial permeability. Mol Cell Biol 2007, 27:6323-6333.

7. DerMardirossian C, Rocklin G, Seo JY, Bokoch GM: Phosphorylation of RhoGDI by Src regulates Rho GTPase binding and cytosol-membrane cycling. Mol Biol Cell 2006, 17:4760-4768.

8. Unwin RD, Sternberg DW, Lu Y, Pierce A, Gilliland DG, Whetton AD: Global effects of BCR/ABL and TEL/PDGFRbeta expression on the proteome and phosphoproteome: identification of the Rho pathway as a target of BCR/ ABL. J Biol Chem 2005, 280:6316-6326.

9. Sun $G$, Kemble DJ: To $C$ or not to $C$ : direct and indirect redox regulation of Src protein tyrosine kinase. Cell Cycle 2009, 8:2353-2355.

10. Mehdi MZ, Pandey NR, Pandey SK, Srivastava AK: H2O2-induced phosphorylation of ERK $1 / 2$ and PKB requires tyrosine kinase activity of insulin receptor and c-Src. Antioxid Redox Signal 2005, 7:1014-1020.

11. Rosado JA, Redondo PC, Salido GM, Gomez-Arteta E, Sage SO, Pariente JA: Hydrogen peroxide generation induces pp60src activation in human platelets: evidence for the involvement of this pathway in storemediated calcium entry. J Biol Chem 2004, 279:1665-1675.

12. Sangrar W, Gao Y, Scott M, Truesdell P, Greer PA: Fer-mediated cortactin phosphorylation is associated with efficient fibroblast migration and is dependent on reactive oxygen species generation during integrinmediated cell adhesion. Mol Cell Biol 2007, 27:6140-6152.

13. Wang Z, Thurmond DC: Differential phosphorylation of RhoGDI mediates the distinct cycling of $\mathrm{Cdc} 42$ and Rac1 to regulate second-phase insulin secretion. J Biol Chem 2010, 285:6186-6197.

14. Hao QL, Ferris DK, White G, Heisterkamp N, Groffen J: Nuclear and cytoplasmic location of the FER tyrosine kinase. Mol Cell Biol 1991, 11:1180-1183.

15. Rosato R, Veltmaat JM, Groffen J, Heisterkamp N: Involvement of the tyrosine kinase fer in cell adhesion. Mol Cell Biol 1998, 18:5762-5770.

16. Voisset E, Lopez S, Chaix A, Georges C, Hanssens K, Prebet T, Dubreuil P, De Sepulveda P: FES kinases are required for oncogenic FLT3 signaling. Leukemia 2010, 24:721-728.

17. Itoh T, Hasegawa J, Tsujita K, Kanaho Y, Takenawa T: The tyrosine kinase Fer is a downstream target of the PLD-PA pathway that regulates cell migration. Sci Signal 2009, 2:ra52.

18. Kweon SM, Cho YJ, Minoo P, Groffen J, Heisterkamp N: Activity of the Bcr GTPase-activating domain is regulated through direct protein/protein interaction with the Rho guanine nucleotide dissociation inhibitor. J Biol Chem 2008, 283:3023-3030.

19. Hao QL, Heisterkamp N, Groffen J: Isolation and sequence analysis of a novel human tyrosine kinase gene. Mol Cell Biol 1989, 9:1587-1593.

doi:10.1186/1471-2091-11-48

Cite this article as: Fei et al:: The Fer tyrosine kinase regulates interactions of Rho GDP-Dissociation Inhibitor $\alpha$ with the small GTPase Rac. BMC Biochemistry 2010 11:48.

\section{Submit your next manuscript to BioMed Central and take full advantage of:}

- Convenient online submission

- Thorough peer review

- No space constraints or color figure charges

- Immediate publication on acceptance

- Inclusion in PubMed, CAS, Scopus and Google Scholar

- Research which is freely available for redistribution

Submit your manuscript at www.biomedcentral.com/submit 